\title{
Rare Case of Choking Death by Dispersible Tablet: A Case Report
}

Shailendra G Dhawane ${ }^{1}$, Nitin S Barmate ${ }^{2^{*}}$, Sharad V Kuchewar ${ }^{3}$ and Ranjit M Tandle ${ }^{4}$

${ }^{1}$ Department of Forensic Medicine, Govt Medical College, Nagpur, Maharashtra, India

${ }^{2}$ Department of Forensic Medicine, Pt. J N M Medical College, Raipur, Chhattisgarh, India

${ }^{3}$ Department of Forensic Medicine, Govt Medical College, Yavatmal, Maharashtra, India

${ }^{4}$ Department of Forensic Medicine, D V Vikhe Patil Medical college, Ahmed Nagar, Maharashtra, India

"Corresponding author: Nitin S Barmate, Asst. Professor, Department of Forensic Medicine, Pt. J N M Medical College, Raipur, Chhattisgarh - 492001, India, Tel: 918889083686; E-mail: nitinsforensic@gmail.com

Rec date: Oct 07, 2014 Acc date: July 08, 2015 Pub date: July 13, 2015

Copyright: (c) 2015 Dhawane GS, et al. This is an open-access article distributed under the terms of the Creative Commons Attribution License, which permits unrestricted use, distribution, and reproduction in any medium, provided the original author and source are credited.

\begin{abstract}
Choking is a form of asphyxia caused by an obstruction within the air passage. Aspiration of a foreign body is common in children and cause upper airway obstruction leading to significant morbidity and mortality. In most of the cases, death is due to mechanical obstruction of airway, in some cases, death may be due to reflex spasm of respiratory tract or by neurogenic cardiac arrest. Deaths due to choking are relatively rare occurrence.
\end{abstract}

\section{Introduction}

Choking is a form of asphyxia caused by an obstruction within the air passage. Complete obstruction of windpipe is not required to cause death by choking [1]. The deaths typically are peaked at the extreme of age with young children and the elderly having the greatest rate of fatal choking [2]. Aspiration of a foreign body is common in children and cause upper airway obstruction leading to significant morbidity and mortality [3]. In most of the cases, death is due to mechanical obstruction of airway. However, in some cases, death may be due to reflex spasm of respiratory tract or may be brought about by neurogenic cardiac arrest [4]. Here in we are reporting a case report of choking death following oral administration of Nimesulide Dispersible Tablet in a young child suffering from pyrexia.

\section{Case}

A 2 year old female child had history of fever since one day. An Ayurveda practitioner prescribed antipyretic Nimesulide Dispersible tablet and multivitamin tonic to her. The father of deceased child put intact Dispersible tablet in her mouth on the tongue by extending the neck and pouring a small quantity of water thereafter. There was a bout of single vomiting followed by sudden collapse immediately after it. She was immediately rushed to our hospital where she was declared brought in dead condition.

\section{On Autopsy examination}

On the same day of the incidence the deceased was brought for medico legal autopsy at our hospital. On external examination-The deceased was average built with well-developed rigor mortis in whole body. Post-mortem lividity was seen over back and was fixed.

No evidence of any external or internal injuries. No sign of asphyxia. Small quantity of whitish semisolid sticky particles of dispersible tablet was found adherent near the root of tongue (Figures 1 and 2), over pharynx, posterior surface of epiglottis (Figures 1 and 2), vocal cords (Figures 1 and 2), with the evidence of trickling the particles in the trachea (Figure 3), up to the right bronchus (Figure 4).
The respiratory track was not completely occluded. Brain- pale, Lungs - edematous and congested. Stomach contents - 100gm of yellowish food material containing dal and rice (Figure 5), with no evidence of regurgitation in the respiratory passage. No organic pathology was found on gross examination and histopathalogical examination. "Choking" was labelled as cause of death.

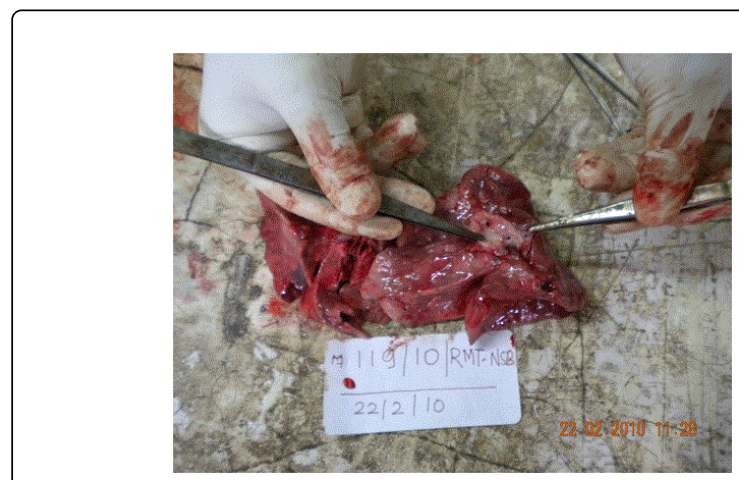

Figure 1: Sticky tablet particle in right bronchus

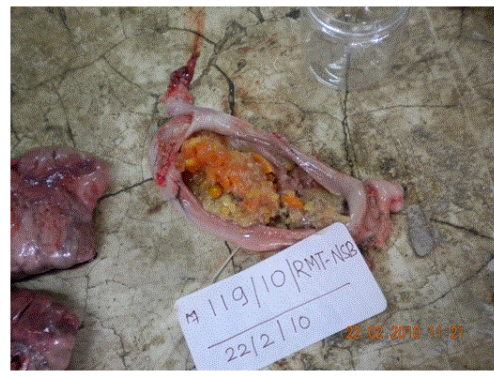

Figure 2: Stomach Contents 


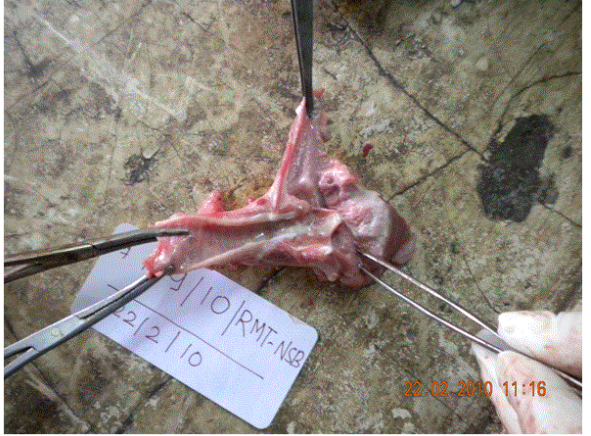

Figure 3: Tablet Particle Adherent over Epiglottis, Vocal cord, Trachea

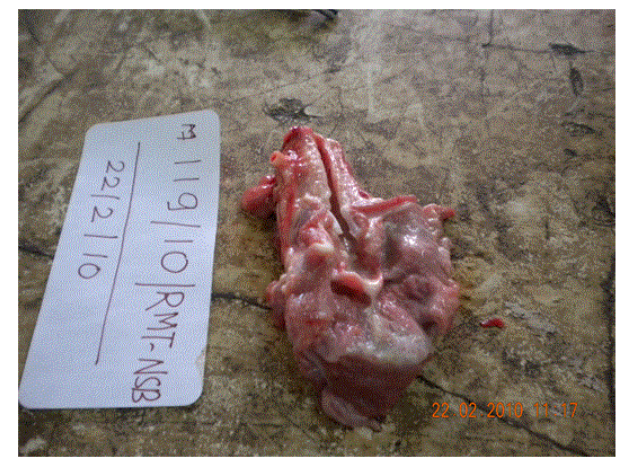

Figure 4: Tablet particle adherent to root of tongue and epiglottis.

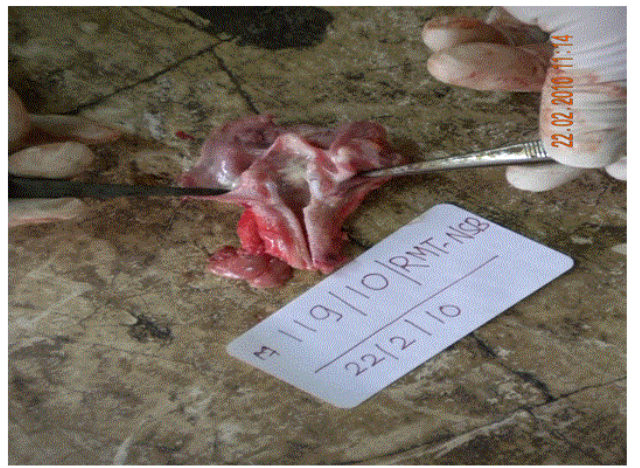

Figure 5: Tablet Particle in Tracheal Lumen

\section{Discussion}

Choking is commonly seen in the very young, elderly, psychiatric patients or in a case where the ability to swallow or masticate is severely impaired like the infirm, acute alcohol intoxication etc. [5]. Nimesulide which is a selective cox-2 inhibitor and a non-steroidal anti-inflammatory drug (NSAID) contains analgesic and antipyretic properties [6].
The dispersible tablets that are given to the patients orally after dissolving them in small quantity of water [7]. The tablet unfortunately, in this case the deceased father directly put the intact dispersible tablet in her mouth and pour water. During the course of dispersion the tablet particles got adherent in the oral cavity and respiratory passage. Stimulation of pharynx by the tablet particles might have resulted in sudden onset of vomiting.

Accidental choking is an important cause of death in young children [8]. Death in choking could be due to asphyxia, vagal inhibition or laryngeal spasm. Complete blockage of the air passage is not required to cause death by choking. Death can occur from laryngeal spasm, when small objects block the lumen only partially [5].

Choking usually causes death by mechanical asphyxia there are undoubted occasions when immediate result of choking is vagal inhibition and sudden death. The absence of other signs makes the diagnosis difficult especially when the victim is an infant and quantity of material in the air passage is small [9].

Smaller foreign bodies lodge in larynx may not obstruct the airway completely, induced laryngeal spasm. Sudden reflex neurogenic cardiovascular failure occurs in laryngeal obstruction. The cardiovascular failure induced by reflex parasympathetic cardiac inhibition. Reflex parasympathetic cardiac inhibition may also occur when foreign body impacted in bronchus with striking absence of asphyxia sign [10].

In the present case, tablet particles adherent to pharynx, larynx with evidence of trickling the particles in the trachea up to the right bronchus with absence of asphyxia signs, histopathalogical examination negative for organ pathology, details of circumstances and exclusion of other possible causes favours vagal inhibition due to choking in stated case. The mode of death probably being cardiac arrest by vagal inhibition due to irritation of pharyngeal and laryngeal mucosa caused by tablet particles resulting in stimulation of branches of vagus nerve.

Food material asphyxiation remains a common problem particularly in children between 1 to 3 years of age. These fatal accidents can be prevented by parental education and early recognition and management of the situation [11].

\section{References}

1. Narayan Reddy KS (2010) Essentials Of Forensic Medicine, And Toxicology 29: 324-325.

2. Dolkas L, Stanley C, Smith AM, Vilke GM (2007) Deaths associated with choking in San Diego county. J Forensic Sci 52: 176-179.

3. Qureshi S, Mink R (2003) Aspiration of fruit gel snacks. Pediatrics 111: 687-689.

4. Krishan VIJ (2008) Text Book of Forensic Medicine, And Toxicology, Principal And Practice 4: 157-158.

5. Punia RK (2015) Obstructive asphyxia- A Case Of Choking In A Psychiatric Patient, Journal Of Indian Academy Forensic Medicine 31: 397-398

6. https://En.Wikipedia.Org/Wiki/Nimesulide.

7. www.Unicef.Org/Supply/Index_53571.Html.

8. Dershewitz RA (1996) Causes of choking in children, NEJM Journal Watch General Medicine.

9. Polson CJ, Gee JE, Knight B (1985) The Essentials of Forensic Medicine 4: 461-468.

10. Gordon I, Shapiro HA, Berson SD Forensic Medicine, A Guide To Principles 3: 113-115. 
Citation: Dhawane SG, Barmate NS, Kuchewar SV, Tandle RM (2015) Rare Case of Choking Death by Dispersible Tablet: A Case Report. J Forensic Res S3: 1000S3-003. doi:10.4172/2157-7145.1000S3-003

Page 3 of 3

11. Goren S, Gurkan F, Tirasci Y, Kaya Z, Acar K (2005) Foreign body asphyxiation in children. See comment in PubMed Commons below Indian Pediatr 42: 1131-1133.

This article was originally published in a special issue, entitled: "Analytical Applications in Forensic Sciences", Edited by Harvey Hou, Alabama State University, USA 